\title{
Comparative study of gut microbiota in Tibetan wild asses (Equus kiang) and domestic donkeys (Equus asinus) on the Qinghai-Tibet plateau
}

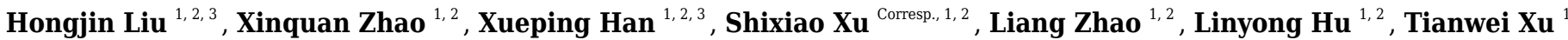 \\ ${ }^{2}$, Na Zhao ${ }^{1,2}$, Xiaoling Zhang ${ }^{1,2,3}$, Dongdong Chen ${ }^{1,2}$, Fuquan $\mathrm{He}^{1,2}$, Xin Chen ${ }^{1,2,3}$ \\ 1 Northwest Institute of Plateau Biology, Chinese Academy of Sciences, Xining, Qinghai Province, China \\ 2 Key Laboratory of Adaptation and Evolution of Plateau Biota, Chinese Academy of Sciences, Xining, Qinghai Province, China \\ 3 University of Chinese Academy of Science, Beijing, China \\ Corresponding Author: Shixiao Xu \\ Email address: sxxu@nwipb.cas.cn
}

Tibetan wild asses (Equus Kiang) are the only wild species of perissodactyls on the Qinghai-Tibet Plateau and appears on the International Union for Conversation of Nature (IUCN) 2012 Red List of threatened species. Therefore, understanding the gut microbiota composition and function of wild asses can provide a theoretical for the situ conservation of wild animals in the future. In this study, we measured the dry matter digestion by the 4 molar hydrochloric acid (4N HCL) acid-insoluble ash method and analyzed the intestinal microbiota of wild asses and domestic donkeys by high-throughput sequencing of the $16 \mathrm{~s}$ rDNA genes in V3-V4 regions. The results showed that the dry matter digestion in wild asses was significantly higher than in domestic donkeys $(P<0.05)$. No significant difference in alpha diversity was detected between these two groups. Beta diversity showed that the bacterial community structure of wild asses was acutely different from domestic donkeys. At the phylum level, the two dominant phyla Bacteroidetes and Firmicutes in wild asses were significantly higher than that in domestic donkeys. At the genus level, Ruminococcaceae_NK4A214, Phascolarctobacterium, Coprostanoligenes_group, Lachnospiraceae_XPB1014_group and Akkermansia in wild asses were significantly higher than in domestic donkeys. Moreover, statistical comparisons showed that 40 different metabolic pathways exhibited significant differences. Among them, 29 pathways had richer concentrations in wild asses than domestic donkeys, mainly included amino acid metabolism, carbohydrate metabolism, and energy metabolism. Of note, network analysis showed that wild asses harbored a relatively more complex bacterial network than domestic donkeys, possibly reflecting the specific niche adaption of gut bacterial communities through species interactions. The overall results indicated that wild asses have advantages over domestic donkeys in dry matter digestion, gut microbial community 
composition and function, and wild asses have their unique intestinal flora to adapt high altitudes on the Qinghai-Tibet plateau. 
1 Comparative Study of Gut Microbiota in Tibetan wild

2 asses (Equus Kiang) and domestic donkeys (Equus

\section{Asinus) on the Qinghai-Tibet plateau}

4 Hongjin Liu ${ }^{1,2,3}$, Xinquan Zhao ${ }^{1,2}$, Xueping $\mathrm{Han}^{1,2,3}$, Shixiao $\mathrm{Xu}^{1,2}$, Liang $\mathrm{Zhao}^{1,2}$, Linyong $\mathrm{Hu}^{1,2}$,

5 Tianwei Xu ${ }^{1,2}$, Na Zhao ${ }^{1,2}$, Xiaoling Zhang ${ }^{1,2,3}$, Dongdong Chen ${ }^{1,2}$, Fuquan $\mathrm{He}^{1,2}$, Xin Chen ${ }^{1,2,3}$.

$6{ }^{1}$ Northwest Institute of Plateau Biology, Chinese Academy of Sciences, Xining, Qinghai

7 Province, China

$8{ }^{2}$ Key Laboratory of Adaptation and Evolution of Plateau Biota, Chinese Academy of Sciences,

9 Xining, Qinghai Province, China

$10{ }^{3}$ University of Chinese Academy of Science, Beijing, China.

12 Corresponding Author:

13 Shixiao $\mathrm{Xu}$,

14 No. 23 Xining Road, Chengxi District, Xining, Qinghai Province, 810008, China

15 Email address: sxxu@nwipb.cas.cn

\section{Abstract}

Tibetan wild asses (Equus Kiang) are the only wild species of perissodactyls on the Qinghai-Tibet

Plateau and appears on the International Union for Conversation of Nature (IUCN) 2012 Red List of threatened species. Therefore, understanding the gut microbiota composition and function of wild asses can provide a theoretical for the situ conservation of wild animals in the future. In this study, we measured the dry matter digestion by the 4 molar hydrochloric acid (4N HCL) acidinsoluble ash method and analyzed the intestinal microbiota of wild asses and domestic donkeys by high-throughput sequencing of the 16s rDNA genes in V3-V4 regions. The results showed that the dry matter digestion in wild asses was significantly higher than in domestic donkeys $(P<0.05)$. 
27 showed that the bacterial community structure of wild asses was acutely different from domestic 28 donkeys. At the phylum level, the two dominant phyla Bacteroidetes and Firmicutes in wild 29 asses were significantly higher than that in domestic donkeys. At the genus level, Ruminococcaceae_NK4A214, Phascolarctobacterium, Coprostanoligenes_group, Lachnospiraceae_XPB1014_group and Akkermansia in wild asses were significantly higher than 32 in domestic donkeys. Moreover, statistical comparisons showed that 40 different metabolic 33 pathways exhibited significant differences. Among them, 29 pathways had richer concentrations 34 in wild asses than domestic donkeys, mainly included amino acid metabolism, carbohydrate 35 metabolism, and energy metabolism. Of note, network analysis showed that wild asses harbored a relatively more complex bacterial network than domestic donkeys, possibly reflecting the specific

37 niche adaption of gut bacterial communities through species interactions. The overall results 38 indicated that wild asses have advantages over domestic donkeys in dry matter digestion, gut microbial community composition and function, and wild asses have their unique intestinal flora to adapt high altitudes on the Qinghai-Tibet plateau.

Abbreviation key: QTP: Qinghai-Tibet Plateau; TWAs: Tibetan wild asses; NPDDs: natural pasture domestic donkeys; 4N HCL: 4 molar hydrochloric acid; AIA: acid-insoluble ash; Avgk: average degree; AvgCC: average clustering coefficient; CB: centralization of betweenness; D: density.

\section{Introduction}

Low temperatures and hypoxia render the Qinghai-Tibet Plateau (QTP) an extremely harsh environment for the survival of mammalian species. The Tibetan wild asses (Equus kiang)—order Perissodactyla, family Equidae, genus Equus - is considered a unique species and the only wild species of perissodactyls to inhabit the QTP (Zheng \& Gao 2000), and appears on the International 
50 Union for Conversation of Nature (IUCN) 2012 Red List of threatened species. Extensive research

51 has been conducted to promote the conservation of this species (Dong et al., 2015; Guo et al.,

52 2018). Domestic donkeys (Equus asinus) — also of the order Perissodactyla, family Equidae, genus

53 Equus - are the subspecies of wild ass (Moehlman, 2002) and were introduced from regions of

54 China at lower elevations, adapting to life in this harsh environment and becoming the livestock

55 of the nomadic Tibetan people to their substantial economic benefit. The intestines of Tibetan wild

56 asses (TWAs) and domestic donkeys contain complex microorganisms that play an important role

57 in converting indigestible plants into the energy needed for host growth, serving a bridge between

58 forage digestion and host energy absorption. Therefore, the study of intestinal microorganisms is

59 of great significance for revealing host immunity, nutrient metabolism, energy absorption and wild

60 animal conservation.

61 Numerous scientific reports suggest that gut microbiota constitute a complex ecosystem

62 involving a symbiotic relationship with the host that plays an important role in the host's

63 performance, health and disease, nutrient absorption, and metabolic status (Hintz \& Cymbaluk

64 1994; O'Hara \&Shanahan 2006; Sekirov et al., 2010; Wardwell et al., 2011). To date, this research

65 has focused mainly on humans, ruminants, marine organisms, and some monogastric animals to

66 investigate the factors that affect intestinal microbiota, gut microbes and health, and gut microbes

67 and nutritional metabolism (Bian et al., 2016; Sekirov et al., 2010; Shepherd et al., 2012). As

68 herbivores, donkeys utilize forage nutrition by microorganism fermentation in the gastrointestinal

69 tract. Zhao et al. (2016) studied bacterial diversity in the ceca of Xinjiang donkeys and found that

70 Ruminococcaceae and Lachnospiraceae played a key role in digesting roughage feed. Due to the

71 difficulty of obtaining samples from wild protected animals, research on gut microbes in TWAs is

72 limited. A recent study compared the gut microbiota composition of wild and captive TWAs, 
73 revealing that captivity reduced intestinal microbial diversity and increased the risk of epidemics,

74 thereby negatively impacting the health of wildlife (Gao et al., 2019). However, few studies have

75 compared the intestinal microbial composition and function of TWAs and natural pasture domestic

76 donkeys (NPDDs) on the QTP.

77 As members of the same genus_Equus_- TWAs and NPDDs provide ideal candidates for

78 the study of gut microbiota. Therefore, we used high-throughput sequencing of the V3-V4 regions

79 of the 16s rRNA gene to investigate the composition and predict the metabolic pathways and

80 functions of the gut microbes in TWAs and NPDDs. We hypothesized that TWAs were superior

81 to NPDDs in terms of the composition and function of their intestinal flora. We think our study is

82 of importance for the study of intestinal flora adaptability of Tibetan wild asses at the high altitude.

\section{Materials and Methods}

84 In this study, all animal care procedures were consistent with the guidelines from the Institution

85 of Animal Care and Ethics Committee of the Northwest Institute of Plateau Biology, Chinese

86 Academy of Sciences (NWIPB20160302), besides this project was carried out with the permission

87 of local government (Qinghai provincial sciences \& technology department). Chaoyuan Zhang the

88 deputy director of the China Qinghai provincial science \& technology department and Xinquan

89 Zhao the academic dean of Northwest Institue of Plateau Biology Chinese Academy of Sciences

90 gave these verbal authorizations to allow us to carry out the experiment here. So official documents

91 and field permits are not required.

\section{Sample collection}

93 A total of 30 fecal samples were obtained from two different sites. The TWAs group included

9415 fecal samples from a swamp meadow $\left(101^{\circ} .06^{\prime} \mathrm{E}, 38^{\circ} .37^{\prime} \mathrm{N}\right)$ in Qumarleb County (Yushu

95 Prefecture, Qinghai Province, China) at an altitude of $4300 \mathrm{~m}$ in August 2017; the NPDD group 
96 included 15 fecal samples from an alpine meadow $\left(94^{\circ} 48^{\prime} \mathrm{E}, 34^{\circ} .99^{\prime} \mathrm{N}\right)$ in Menyuan County (Haibei

97 Prefecture, Qinghai Province, China) at an altitude of $3200 \mathrm{~m}$ in August 2017. All of the laboratory

98 animals were healthy and none of the donkeys had been administered antibiotics within the past

99 three months. The fecal samples (approximately $2 \mathrm{ml}$ each) were collected and transferred into

100 separate sterilized tubes and stored immediately in liquid nitrogen for DNA extraction. The forage

101 samples were collected from quadrats $(50 \mathrm{~cm} \times 50 \mathrm{~cm})$ of grass on which the animals grazed.

102 Twenty quadrats greater than $10 \mathrm{~m}$ apart were placed randomly to investigate the dominant species

103 and collect the ground herbage. A total of 20 forage samples were collected from these two regions

104 (10 per region). Forage samples were dried in a $65^{\circ} \mathrm{C}$ oven for $24 \mathrm{~h}$, then ground through a $1-\mathrm{mm}$

105 sieve and stored in a vacuum dryer for nutritional analysis.

106 Determination of plant nutrient composition

107 Total N was measured using the Kjeldahl method; the crude protein content was calculated as 6.25

$108 \times \mathrm{N}($ Method No. 984.13); the ether extract (EE) was measured using the Soxhlet system (Method

109 No. 954.02); the acid detergent fiber (ADF) and neutral detergent fibre (NDF) were analyzed using

110 the method described by Soest et al. (1991) (Soest et al., 1991); the non-fibrous carbohydrate

111 (NSC) was calculated as follows:NSC (\%) = $100-\mathrm{Ash}(\%)-\mathrm{EE}(\%)-\mathrm{NDF}(\%)-\mathrm{CP}(\%)$. The

112 nutrition of the herbage and dominant species are shown in Table 1.

113 Acid-insoluble ash to determine dry matter digestibility

114 The dry matter digestibility was measured by using a modified method of 4N-HCL acid-insoluble

115 ash (AIA) (Van \& Young, 1997). Briefly, 5 gram crushed forage and 5 gram dried excreta are

116 boiled in $50 \mathrm{ml} 4 \mathrm{~N}$ for thirty minutes; the slurry is then filtered by quantitative filtered paper

117 (NEWSTAR $^{\circledR}$, Hangzhou, China, $\Phi 12.5 \mathrm{~cm}$ ) and the residue washed free of acid using hot

118 distilled water untilled the elute is neutral; the residue and the filter paper were ashed directly in 
119 crucible at $600^{\circ} \mathrm{C}$ for 12 hours in electric muffle furnace (Beijing zhongxing weiye instrument co.

120 LTD, KSW-6-12); After the residue and filter paper complete combustion, the crucible and ash

121 were placed in a dryer to cool to room temperature and then weighed on an analytical balance. The

122 equation used to calculate dry matter digestibility was as follows (Kavanagh et al., 2001):

123 Dry matter digestibility $=100-100 \times$ AIA in herbage / AIA in faeces.

\section{DNA extraction and purification}

125 Microbial DNA was extracted from stool samples using the E.Z.N.A ${ }^{\circledR}$ Stool DNA Kit (Omega 126 Bio-Tek, Norcross, GA, USA) according to the manufacturer's protocols. DNA quality was 127 assessed via 2\% agarose gel electrophoresis and metagenomic DNA concentrations were 128 determined with a NanoDrop ${ }^{\mathrm{TM}} 2000$ (Thermo Fisher Scientific, Waltham, MA, USA). The 16S

129 rDNA V3-V4 regions of the ribosomal RNA gene were then amplified by PCR ( $95^{\circ} \mathrm{C}$ for $2 \mathrm{~min}$,

130 followed by 27 cycles at $98^{\circ} \mathrm{C}$ for $10 \mathrm{~s}, 62^{\circ} \mathrm{C}$ for $30 \mathrm{~s}$, and $68^{\circ} \mathrm{C}$ for $30 \mathrm{~s}$, with a final extension

131 at $68{ }^{\circ} \mathrm{C}$ for $10 \mathrm{~min}$ ) using the following primers: 341F, CCTACGGGNGGCWGCAG; 806R,

132 GGACTACHVGGGTATCTAAT. The 5'-end of 314F primer includes 8-bp unique barcodes,

133 which were used to split each sample. PCR reactions were performed in triplicate with a $50 \mu 1$

134 mixture containing $5 \mu \mathrm{l}$ of $10 \times \mathrm{KOD}$ buffer, $5 \mu \mathrm{l}$ of $2.5 \mathrm{mM}$ dNTPs, $1.5 \mu \mathrm{l}$ of each primer $(5 \mu \mathrm{m})$,

$1351 \mu \mathrm{l}$ of KOD polymerase, and $100 \mathrm{ng}$ of template DNA. The PCR products were detected by 136 electrophoresis on a 2\% agarose gel and purified using the AxyPrep DNA Gel Extraction Kit 137 (Axygen Biosciences, Union City, CA, USA) according to the manufacturer's instructions and 138 quantified using QuantiFluor ${ }^{\circledR}$-ST (Promega, Madison, WI, USA). The purified amplicons were 139 then pooled equimolarly for paired-end sequencing on an Illumina (San Diego, CA, USA) HiSeq 
1402500 platform (Guangzhou Gene Denovo Biotechnology Co Ltd Guangzhou, China) using

141 standard protocols.

142 Sequencing and processing

143 To get clean paired-end reads, the raw reads were filtered to remove reads containing greater than

$14410 \%$ unknown nucleotides (Ns) and fewer than $80 \%$ of base calls with quality scores less than 20 .

145 FLASH version 1.2.11 was used to merge paired-end reads as raw tags with a minimum overlap

146 of $10 \mathrm{bp}$ and mismatch error rates of 2\% (Magoc \& Salzberg, 2011). The noisy raw tag sequences

147 were filtered by QIIME (version 1.9.1) to obtain high-quality, clean tags (Caporaso et al., 2010).

148 The filter commands mainly including tags interception and length filtering. That is, the raw tags

149 were intercepted from the continuous low-quality value (the default quality threshold is $<=3$ ) to

150 the first low-quality base site of the set length (the default length is 3); filtering out tags data that

151 had a continuous high-quality base length less than $75 \%$ of the length of the tags. The clean tags

152 then underwent chimera detection using the UCHIME algorithm until all chimeras were removed

153 and effective tags were obtained (Edgar et al., 2011). All of the sequences were clustered into

154 operational taxonomic units (OTUs) of " $\geq 97 \%$ " similarity using the UPARSE version 7.0.1001

155 pipeline (Edgar, 2013) and the representative sequences were classified into organisms by

156 applying a naive Bayesian model using the RDP classifier version 2.2 (Wang et al., 2007) based

157 on the SILVA database (version v132) (Pruesse et al., 2007).

\section{Statistical analysis}

159 The taxonomic composition for each cluster was evaluated at the phylum and genus level. Data

160 related to bacterial community were statistically analyzed using SPSS version 17.0 (SPSS, Inc

161 Chicago, IL, USA). The significance of herbage nutritional composition and bacterial taxa was

162 determined using the independent-samples T test. The bacterial community function was predicted 
163 using Tax4Fun software (version 0.3.1) (Asshauer et al., 2015). The significant KEGG metabolic

164 pathways were screened using STAMP software (version 2.1.3), the statistical method was two

165 groups Welch's t-test, the type was two-sided and the confidence interval method was Welch's

166 inverted (0.95). The OTU rarefaction curves were calculated and plotted in QIIME. All alpha

167 diversity was calculated in QIIME and graphed by Origin version 8.0 (OriginLab ${ }^{\circledR}$, Northampton,

168 MA, USA)). The principal coordinates analysis (PCoA) with unweighted UniFrac distance at OTU

169 level was plotted in R version 3.5.0 and metric distance were calculated by Wilcoxon rank sum

170 test. The unweighted pair-group method with arithmetic means (UPGMA) classification tree was

171 plotted by mothur version 1.41.1 (Schloss et al., 2009). Results are reported as means \pm standard

172 error (SE). The effects were considered significant at $P<0.05$.

173 Network analysis and identification of putative keystone taxa in TWAs

174 and NPDDs

175 To understand the interaction of gut microbiota between TWA and NPDD, phylogenetic molecular

176 ecological networks (pMENs) (http://ieg4.rccc.ou.edu/mena) were constructed based on Random

177 Matrix Theory (RMT)-based methods. The protocols of network construction were described

178 previously (Li et al., 2016). Briefly, only the OTUs that were presented in more than half of all

179 samples were selected to in each TWAs and NPDDs gut microbiota genus. In order to compare

180 the topological characteristics of bacteria network between TWAs and NPDDs, the pMENs were

181 calculated with the same threshold (0.76). For each network, in the above website, the window

182 "global network properties" was used to calculate total nodes, total links, positive links, negative

183 links, average degree (avgK), average clustering coefficient (avgCC), centralization of

184 betweenness (CB) and density (D). It was worth noting that a positive correlation might relate to

185 mutualism, commensalism or parasitism, while a negative correlation might result to competition, 
186 predation, etc (Faust \& Raes, 2012). The window "module separation and modularity

187 calculation" was then used to calculate the value of within-module connectivity ( $\mathrm{Zi}$ ) and among 188 modularity connectivity (Pi). Finally, the visualization of these community co-occurrence 189 networks was made with Cytoscape 3.3.0 (Shannon et al., 2003).

190 A module typically contains many nodes, which are tightly connected to each other in one 191 group, while having only few connections outside the group. The values of $\mathrm{Zi}$ and $\mathrm{Pi}$ are indicators 192 of the connectivity of each node, and thus are often used to determine the topological role of these 193 nodes (Deng et al., 2012). According to the valises of $\mathrm{Zi}$ and Pi, these nodes can be classified into 194 four categories (Zhou et al., 2011), including peripherals (In the modules, the OTUs have few 195 outside connections, $\mathrm{Pi}<0.62$ and $\mathrm{Zi}<2.5$ ), connectors (OTUs that connect modules, $\mathrm{Pi}>0.62$ ), 196 network hubs (OTUs that highly connected within entire network, $\mathrm{Pi}>0.62$ and $\mathrm{Zi}>2.5$ ) and 197 module hubs (OTUs that highly connected within modules, $\mathrm{Zi}>2.5$ ). Connectors, network hubs 198 and module hubs were considered as the putative keystone taxa in a microbial community ( $L i$ et 199 al., 2017). The keystone of gut microbiota in TWAs and NPDDs was identified based on the above 200 values.

201 Results

202 Analysis of food nutrition composition and dry matter digestibility 203 between TWAs and NPDDs

204 As shown in Table1, the mainly plant-based diet for TWAs were Kobresia tibetica and Kobresia 205 pygmaea, while for NPDDs were Kobresia humilis and Kobresia capillifolia. There was no 206 significant difference in nutrient composition except ash content $(P<0.05)$. In addition, the dry 207 matter digestibility was measured by acid-insoluble ash method. As shown in Table 2, AIA in the 
208 faces of NPDDs were significantly higher than TWAs group $(P<0.05)$, whereas, dry matter

209 digestibility in NPDDs were significantly lower than TWAs group $(P<0.05)$.

210 Sequencing and classification

211 Illumina sequencing yielded 2,540,672 raw reads of $16 \mathrm{~S}$ gene sequences. After quality filtering, a

212 total of 2,395,867 effective tags were obtained (Table S1). As shown in Fig. S1, 12,923

213 operational taxonomic units (OTUs) at a cutoff of 97\% similarity were generated. Among these

214 OTUs, the number of unique OTUs in TWAs and NPDDs was 2948 and 2816, respectively, and

2157159 OTUs were shared by all samples, accounting for $55.40 \%$ of the total OTUs.

216 Diversity analysis of gut microbiota in Tibetan wild ass and domestic

217 donkey

218 To determine alpha diversity, we calculated the Chao1, ACE, observed species estimates and the

219 Shannon diversity index. As shown in Fig. 1 and Fig S2, no significant differences were found in

220 these two groups for the Shannon index, Chao1, ACE estimates, the number of observed species

221 and Faith's phylogenetic diversity (PD). The OTU level rarefaction curves of diversity estimators

222 reached a plateau at the minimum sequence number of 50,000 tags (Fig. S3). With regard to beta

223 diversity, PCoA analysis showed that the two groups of bacterial communities differed

224 significantly (Fig. S4A) $(P=3.02 E-05)$. To assess the overall similarity, UPGAMA with

225 classification tree analysis was conducted as shown in Fig. S4B. It is obvious that the bacterial 226 community of TWAs was completely different from that of NPDDs.

227 Significance analysis of gut bacterial community abundance between 228 TWAs and NPDDs 
229 At the phylum level illustrated in Fig. 2A, Bacteroidetes, Firmicutes, Verrucomicrobia, and

230 Fibrobacteres constituted the four dominant phyla in all samples. The average ratio of

231 Firmicutes/Bacteroidetes was 0.80 and 0.74 , respectively. The significance analysis of the top 10

232 phyla of microbial communities between TWAs and NPDDs is shown in Fig. 2 B and Table S2.

233 The relative abundance of Bacteroidetes, Firmicutes, Cyanobacteria, and Synergistetes in TWDs

234 was significantly higher than in NPDDs, whereas Lentisphaerae in TWAs was significantly lower

$235(P<0.05)$. No sinificant differences were detected for the relative abundances of Verrucomicrobia,

236 Fibrobacteres, Spirochaetaes, Proteobacteria, and Planctomycetes between the two groups $(P>$

$2370.05)$.

238 At the genus level, a total of 15 genera with proportions above $1 \%$ were detected, as shown

239 in Fig. 3A. The Rickenllaceae_RC9_gut group, Fibrobacter, and Treponema_2 accounted for the

240 largest proportions in all samples. Differentiation analysis of the 15 genera is presented in Fig. 3B

241 and Table S3. The proportion of Ruminococcaceae_NK4A214, Phascolarctobacterium,

242 Coprostanoligenes_group, Lachnospiraceae_XPB1014_group, and Akkermansia in TWAs was

243 significantly higher than in NPDDs, whereas the proportion of the

244 Lachnospiraceae_AC2044_group in NPDDs was significantly higher than in TWAs $(P<0.05)$.

245 No remarkable difference was detected in the relative abundance of the

246 Rickenellaceae_RC9_gut_group, Fibrobacter, Treponema, Ruminococcaceae_UCG-010,

247 Christensenellaceae_R-7_group, Prevotellaceae_UCG-001, Anawrovorax,

248 Prevotellaceae_UCG-003, and Prevotellaceae_UCG-004 between the two groups $(P>0.05)$.

249 To study the main differences in gut microbiota between groups TWA and NPDD, linear

250 discriminant analysis (LDA) combined effect size measurements (LEfSe) were used to identify

251 the discriminative features of bacteria in the TWAs and NPDDs (Fig. S5). The taxonomic units of 
252 Campylobacter, Campylobacteraceae, Campylobacterales, Epsilonproteobacteria, and

253 Campylobacter_hyointestinalis_subsp_hyointestinalis, which belong to the phylum

254 Proteobacteria, were significantly enriched and revealed different biomarkers in TWAs, whereas

255 no specific taxonomic units were enriched in NPDD.

256 Predicted metabolic pathways and functions of the bacterial microbiota

257 between TWAs and NPDDs

258 A total of six types of biological functional pathways in KEGG level 1 were detected (Fig. 4A).

259 The metabolism pathways had the highest relative abundance, with more than $60 \%$ of the total

260 reads in each group. At KEGG level 2, a total of 37 metabolic pathways were detected in the gut

261 samples (Table S4). As shown in Fig. 4B, 18 gene families were remarkably different and the

262 relative abundance of amino acid metabolism, energy metabolism, glycan biosynthesis, and the

263 biosynthesis of other secondary metabolites in TWAs were significantly higher than in NPDDs $(P$

$264<0.05)$. As for membrane transport and cell motility, NPDDs had higher expression abundances

265 than TWAs $(P<0.05)$. At KEGG level 3, the principal component analysis and partial least squares

266 discriminant analysis revealed clear differences between the two groups (Fig. 5). As shown in

267 Table S5, 40 metabolite pathways were significantly different (VIP $>1$ and $P<0.05$ ), of which

268 ten metabolite pathways belonging to amino acid metabolism were enriched in TWAs $(P<0.05)$.

269 A total of ten metabolic pathways belonged to carbohydrate metabolism of which seven

270 metabolites were enriched in TWAs, whereas the remaining three metabolites (starch and sucrose

271 metabolism, amino sugar and nucleotide sugar metabolism, and lipopolysaccharide biosynthesis)

272 were enriched in NPDDs $(P<0.05)$. In addition, five metabolic pathways belonged to the

273 metabolism of cofactors and vitamins, of which two metabolites were higher in TWAs. For the

274 remaining 15 metabolic pathways, 11 had a higher relative abundance in TWAs. 


\section{Network analysis of bacterial communities and putative keystone taxa}

276 As shown in Fig. 6, when using the same threshold (0.76), a total of 207 and 104 links were

277 identified for TWA and NPDD. Notably, for TWAs, the percentage of positive correlations (149

278 out of 207) was dominant, while negative correlations (55 out of 104) were the predominant in

279 NPDDs. Interestingly, we found the values of total links, avgK and D were higher in TWAs than

280 those in NPDDs, indicating a relatively simpler network in NPDDs. The lower avgCC in NPDD

281 indicated that the bacterial network was mainly loose node groups. Moreover, TWA had a higher

282 level of CB than NPDD, implying that TWA harbored a higher frequency of centralized.

283 The putative keystone genus in bacterial networks was identified based on the Pi and $\mathrm{Zi}$ values

284 (Table 3). Three key module hubs and two connectors were identified in NPDD. Among these

285 OTUs, module hubs were affiliated with Firmicutes, Cyanobacteria and unclassified bacteria, and

286 the connectors were Anaerovibrio and unclassified bacteria. In contrast, four connectors and no

287 module hubs were identified in TWAs. Among these OTUs, two connectors were affiliated with

288 Firmicutes and Actinobacteria and the others were the genus Oscillospira and Arthrobacter.

289 Notably, these keystone genera in network showed relatively low abundances in gut bacterial 290 communities.

\section{Discussion}

292 TWAs and NPDDs are herbivores species on the QTP, however, scant research has been conducted 293 on their gut microbiota diversity and composition. To the best of our knowledge, this is the first 294 study to compare the gut microbiota composition and function of TWAs and NPDDs. We found 295 that the Shannon-Wiener index and ACE and Chaol estimates, as well as the number of observed 296 species between TWAs and NPDDs were not significantly different, suggesting that altitude and 297 grassland types did not affect the alpha diversity of gut microbes. A previous study had shown that 
298 diet was one of the factors that affected gut microorganism diversity (Daniel et al., 2014). In our 299 study, there was no significant difference in herbage nutrient composition between TWAs and 300 NPDDs in different meadow types (swamp meadow and alpine meadow, respectively), which was 301 consistent with the results of the alpha diversity analysis. In terms of biological classification, 302 TWAs and NPDDs are animals of the same genus but different species; however, there was no 303 significant difference in the alpha diversity of gut microbes, suggesting that species differences 304 did not affect the alpha diversity of bacteria community, similar result was also found in the 305 previous study on wild and domestic yak (Liu et al., 2019). As to beta diversity, PCoA results 306 indicated that the composition of gut microbiota was significantly different in these two groups.

307 For a better understanding of the similarity of their gut microbiota, UPGMA methods were applied 308 and the diagram revealed that the fecal microorganism communities in TWAs occupied their own 309 phylogenetic tree branch which clearly distinguished from the fecal microorganisms in NPDDs.

310 Therefore, we concluded that species, altitude, and meadow type can affect the intestinal bacterial 311 structure; however, the determination of which of these played the leading role in shaping intestinal 312 flora requires further studies.

313 To assess the composition of the bacteria community, we first analyzed at the phylum level.

314 In our study, Bacteroidetes was the most abundant phylum followed by Firmicutes in both TWAs 315 and NPDDs, constituting more than $80 \%$ of the total bacteria content. This has been confirmed by 316 former studies on intestinal microbial diversity in mammals (Mariat et al., 2009; Middelbos et al.,

317 2010; Sahu \& Kamra 2002), as these microorganisms facilitate the digestion of cellulose and

318 hemicellulose in the diet (Wu et al., 2016). The relative abundance of Bacteroidetes and Firmicutes

319 in TWAs was significantly higher than in NPDDs, indicating that the cellulose and hemicellulose

320 decomposition capacity was stronger in TWAs than in NPDDs under similar forage nutritional 
321 composition, and the higher dry matter digestion capacity in the large intestine in TWAs also

322 supports this claim. Moreover, the ratio of Bacteroidetes and Firmicutes affected energy

323 acquisition and body fat accumulation in humans and mice (Turnbaugh et al., 2006). Previous

324 studies have shown that a decrease in Bacteroidetes was strongly linked to an increase of fat in

325 host tissue in mice and humans (Ruth E. Ley \& Gordon, 2006; Turnbaugh et al., 2006).However,

326 many recent studies have found controversial results. Schwiertz et al. (2010) found that Firmicutes

327 / Bacteroidetes ratio changed in favor of the Bacteroidetes in overweight and obese adults. With

328 reagrad to perissodactyla animals, however, scant research has focused on the relationship between

329 gut microbiota and obesity in TWAs and NPDDs. By observing the body size of TWAs and

330 NPDDs, we speculated that the lean body size of TWAs might be related to the high Bacteroidetes

331 content in their intestinal microbiota. At the genus level, 15 core genera whose relative abundances

332 were more than $1 \%$ were detected in all gut samples; these genera constituted $32.43 \%$ of the total

333 genera reads and were mainly affiliated with the phylum Firmicutes (nine genera) and phylum

334 Bacteroidetes (four genera). The others were affiliated with the phyla Spirochaetes (one genus)

335 and Verrucomicrobia (one genus). These results were also reported in ruminant research on

336 animals such as Yaks and Tibetan sheep (Li et al., 2019; Xue et al., 2017), indicating that although

337 TWAs and NPDDs belonged to the genus Equus and were different from ruminants, their

338 microorganism flora communities were actually quite similar. In addition, an average of $54.43 \%$

339 genera presented as unclassified, which suggested that this group of organisms may be a part of

340 the core bacterial population, warranting further investigation in the field on Equus.

341 Recent studies have shown that changes in gut microbiota composition are associated with

342 host disease (Costa et al., 2012; Qin et al., 2012; Sekirov et al., 2010). Bacteroidetes, a basal

343 microbe, is reported to be the most abundant phylum in healthy people (Eckburg et al., 2005) and 
344 captive TWAs (Gao et al., 2019); a decrease in its relative abundance is associated with chronic

345 diarrhea in humans (Paul B. Eckburg, 2005) and could be a predictor of an animal's health. In our

346 study, it accounted for a large proportion in TWAs (44.12\% of sequences), indicating that TWAs

347 may have a superior capacity to stave off intestinal diseases. At the genus level, Akkermansia

348 accounts for the highest proportion in Verrucomicrobia. Researchers have found that Akkermansia

349 currently contains a single species, namely Akkermansia muciniphila, which is an important

350 probiotic that contributes healthy mucus-related microbiota composition (Belzer \& de Vos, 2012;

351 Liu et al., 2014). In addition, Akkermansia muciniphila can be used as a probiotic to prevent obesity

352 and type 2 diabetes (Everard et al., 2013; Qin et al., 2012). In our research, TWAs had a higher

353 distribution proportion of Akkermansia muciniphila, which may be important to the maintenance

354 of intestinal health; however, further study is necessary to verify this assertion.

355 The metabolites of gut microbiota play a pivotal role in maintaining physiologic and 356 metabolic homeostasis in their hosts (Human Microbiome Project 2012). In the present study, the

357 metagenomic function prediction showed that the two predominant intestinal microbiota in TWAs

358 and NPDDs were related to ABC transporters and the two-component system (Table S6). Previous

359 research (Xiong et al., 2014; Zeng et al., 2017) has confirmed that ABC transporters constitute one

360 of the largest known protein families and are widespread in bacteria, archaea, and eukaryotes, and

361 the two-component system is a signal transduction system that senses developmental and

362 environmental stimuli (Podgornaia \& Laub, 2013). Therefore, it is reasonable that these KOs were

363 found to be in high abundance in the intestinal microbiota of TWAs and NPDDs. Moreover,

364 statistical comparisons showed that 40 different metabolic pathways exhibited significant

365 differences (Table S5). Among them, 29 pathways had richer concentrations in TWAs than

366 NPDDs, mainly amino acid metabolism, carbohydrate metabolism, and energy metabolism. A 
367 large number of various microorganisms inhabit the intestinal tracts of monogastric animals that

368 are symbiotic with the hosts. Gut bacteria produce a range of metabolites that provide nitrogen

369 sources for themselves and amino acids for the hosts through the fermentation of protein and

370 nitrogen compounds. In our study, the high concentration of amino acid metabolic pathways in

371 TWAs implied that in the context of high altitude and forage shortage, the gut microorganisms and

372 hosts coevolved and exploited high bioavailability to efficiently utilize nitrogen compounds in

373 forage, ultimately with microorganisms providing themselves a nitrogen source and in return

374 producing various amino acids to maintain normal life activities for the hosts. Research has

375 revealed that carbohydrates in forage can be fermented by intestinal microorganisms and the

376 metabolites include certain short chain fatty acids (SCFAs) which can stimulate the intestinal

377 mucosa, improve intestinal immunity, and promote energy absorption in the hosts (El Kaoutari et

378 al., 2013; Jha \& Leterme, 2012). In our study, the concentration of carbohydrate metabolic

379 pathways in TWAs had an advantage over NPDDs, which meant that microorganisms in the gut

380 of TWAs could more efficiently promote cellulose and hemicellulose fermentation. As for energy

381 metabolism, we found that the citrate cycle in TWAs was significantly more active than in NPDDs,

382 indicating that the intestinal bacteria of TWAs could provide more energy for their own metabolic

383 activities and were therefore beneficial for microorganism fermentation of forage in the intestine.

384 There are complex interactions in gut microbiota, such as mutualism, competition, prediction

385 and commensalism (Faust \& Raes, 2012). Understanding the interactions and their responses to

386 environmental is an important goal in ecology. However, defining a network structure in microbial

387 communities is especially challenging due to their vast diversity and as-yet uncultivated status.

388 Thus, we used the network analysis to investigate the genus interactions according to their mutual

389 competition (negative correlation) and mutual benefit (positive correlation). In our network 
390 analysis, the TWAs were predominated by higher positive correlations, while NPDDs were

391 predominated by negative correlations. We speculated that plant-based diet in the gut of TWAs

392 was more conductive to fermentation through the more corporation interactions than that of

393 NPDD. In addition, we found that in TWAs, the total links, avgD and D were relatively higher

394 than that of NPDDs, indicating that TWAs harbored a more complex ecological network, mostly

395 also more metabolite. More complex interactions could build a more stable metabolic network

396 environment for the microbial population. Further study is needed to confirm this hypothesis.

397 A lower avgCC revealed that bacterial communities are largely composed of isolated nodes

398 or loosely connected node groups, reflecting lower functional redundancy (Sun et al., 2013). In

399 our study, the avgCC in TWAs was relatively higher than NPDDs, indicating that the latter may

400 harbor lower function redundancy than the former. The previous study had revealed that

401 centralized nodes represent keystone species, which may influence the "information" flow among

402 microbes (Bissett et al., 2013). In addition, these nodes play an important role in connecting many

403 other microbes and are generally considered as important control points in the networks a low level

404 of $\mathrm{CB}$, as a result of a low frequency of centralized nodes (Bissett et al., 2013). Thus, a higher CB

405 in TWAs, indicated that the microorganism had different status in networks, implying that the loss

406 of one or several keystone species may affect the stability of the whole bacterial community. Our

407 result also showed that NPDDs harbored more keystone genus than TWA. Here, a total of three

408 module hubs and six connectors were observed in TWAs (four connectors) and NPDDs (three

409 module hubs and two connectors, respectively). However, none of them was the top 10 most

410 abundant genus. The most abundant module microorganism OTU67 was at the 53th position,

411 which indicated that the keystone genus in network not necessary the dominant microbes in the

412 gut microbial community. However, the topological features are related to sequencing depth and 
413 different data processing. In our study, we only remained the OTUs that were present more than

414 half of all samples, and this data processing may influence the topological features in bacteria

415 network.

\section{Conclusions}

417 This study described the dry matter digestion, compositions, diversity and function of 418 bacterial commubities in Tibetan wild asses and domestic donkeys. Based on 16s rRNA 419 sequencing data, we found that the gut microbiota of TWAs was superior to that of NPDDs in 420 bacterial community composition, function, and potentially high resistance to disease risk under 421 similar forage nutrition intake. In addition, high dry matter digestion ensured wild assess use the 422 forage efficiently in high altitude on the Qinghai-Tibet Plateau. As the only wild species of 423 perissodactyls on the Qinghai-Tibet Plateau, the protection of Tibetan wild asses is of great 424 significance to maintain the balance of the ecosystem and the harmony between human and 425 animals. From the perspective of molecular biology, we studied the difference of gut microbiota 426 between Tibetan wild asses and domestic donkeys. These results may help us understand the 427 assembly of bacteria community in the gut of wild and domestic animals and provided a theoretical 428 basis for the adaptability of the intestinal flora of Tibetan wild asses at high altitudes.

\section{Acknowledgments}

430 We express our sincere gratitude to Dr Qi Li for his warm-hearted help of the research group 431 during the sample collection.

\section{References}

433 Asshauer KP, Wemheuer B, Daniel R, Meinicke P. 2015. Tax4Fun: predicting functional 434 profiles from metagenomic 16S rRNA data. Bioinformatics 31:2882-2884. 
435 Belzer C, de Vos WM. 2012. Microbes inside--from diversity to function: the case of 436 Akkermansia. ISME J 6:1449-1458.

437 Bian G, Ma S, Zhu Z, Su Y, Zoetendal EG, Mackie R, Liu J, Mu C, Huang R, Smidt H, Zhu

438

439

440

441

442

443

444

445

446

447

448

449

450

451

452

453

454

455

456

W. 2016. Age, introduction of solid feed and weaning are more important determinants of gut bacterial succession in piglets than breed and nursing mother as revealed by a reciprocal cross-fostering model. Environ Microbiol 18:1566-1577.

Bissett A, Brown MV, Siciliano SD, Thrall PH. 2013. Microbial community responses to anthropogenically induced environmental change: towards a systems approach. Ecol Lett 16 Suppl 1:128-139.

Caporaso JG, Kuczynski J, Stombaugh J, Bittinger K, Bushman FD, Costello EK, Fierer N, Peña AG, Goodrich JK, Gordon JI, Huttley GA, Kelley ST, Knights D, Koenig JE, Ley RE, Lozupone CA, McDonald D, Muegge BD, Pirrung M, Reeder J, Sevinsky JR, Turnbaugh PJ, Walters WA, Widmann J, Yatsunenko T, Zaneveld J, Knight R. 2010. QIIME allows analysis of high-throughput community sequencing data. Nature methods $7: 335$.

Costa MC, Arroyo LG, Allen-Vercoe E, Stampfli HR, Kim PT, Sturgeon A, Weese JS. 2012. Comparison of the fecal microbiota of healthy horses and horses with colitis by high throughput sequencing of the V3-V5 region of the 16S rRNA gene. PLoS One 7:e41484.

Daniel H, Gholami AM, Berry D, Desmarchelier C, Hahne H, Loh G, Mondot S, Lepage P, Rothballer M, Walker A, Bohm C, Wenning M, Wagner M, Blaut M, SchmittKopplin P, Kuster B, Haller D, Clavel T. 2014. High-fat diet alters gut microbiota physiology in mice. ISME $J$ 8:295-308.

Peer) reviewing PDF | (2020:01:44574:1:1:NEW 29 Feb 2020) 
457 Deng Y, Jiang Y-H, Yang Y, He Z, Luo F, and Zhou J .2012. Molecular ecological network $458 \quad$ analyses. BMC bioinformatics 13:113.

459 Dong S, Wu X, Liu S, Su X, Wu Y, Shi J, Li X, Zhang X, Xu D, Weng J. 2015. Estimation of 460 ecological carrying capacity for wild yak, kiang, and Tibetan antelope based on habitat

461 suitability in the Aerjin Mountain Nature Reserve, China. Acta Ecol Sin 35:7598-7607.

462

463

464

465

466

467

468

469

470

471

472

473

474

475

476

477

Eckburg PB, Bik EM, Bernstein CN, Purdom E, Dethlefsen L, Sargent M, Gill SR, Nelson KE, Relman DA. 2005. Diversity of the human intestinal microbial flora. Science 308:1635-1638.

Edgar RC. 2013. UPARSE: highly accurate OTU sequences from microbial amplicon reads. Nature methods 10: 996-998.

Edgar RC, Haas BJ, Clemente JC, Quince C, Knight R. 2011. UCHIME improves sensitivity and speed of chimera detection. Bioinformatics 27:2194-2200.

El, Kaoutari A, Armougom F, Gordon JI, Raoult D, Henrissat B. 2013. The abundance and variety of carbohydrate-active enzymes in the human gut microbiota. Nature Reviews Microbiology 11:497.

Everard A, Belzer C, Geurts L, Ouwerkerk JP, Druart C, Bindels LB, Guiot Y, Derrien M, Muccioli GG, Delzenne NM, de Vos WM, Cani PD. 2013. Cross-talk between Akkermansia muciniphila and intestinal epithelium controls diet-induced obesity. Proceedings of the National Academy of Sciences 110 (22): 9066-9071.

Faust K, and Raes J. 2012. Microbial interactions: from networks to models. Nature Reviews Microbiology 10:538-550. 
478 Gao H, Chi X, Qin W, Wang L, Song P, Cai Z, Zhang J, Zhang T. 2019. Comparison of the

479 Gut Microbiota Composition between the Wild and Captive Tibetan Wild Ass (Equus $480 \quad$ kiang). J Appl Microbiol 126:1869-1878.

481 Guo X, Shao Q, Li Y, Wang Y, Wang D, Liu J, Fan J, Yang F. 2018. Application of UAV

482

483

484

485

486

487

488

489

490

491

492

493

494

495

496

497

498

499

Remote Sensing for a Population Census of Large Wild Herbivores-Taking the Headwater Region of the Yellow River as an Example. Remote Sensing. Remote Sens 10(7): 1041.

Hintz HF, Cymbaluk NF. 1994. Nutrition of the Horse. Annual Review of Nutrition 14:243-267.

Human. Microbiome Project C. 2012. Structure, function and diversity of the healthy human microbiome. Nature 486:207-214.

Jha R, Leterme P. 2012. Feed ingredients differing in fermentable fibre and indigestible protein content affect fermentation metabolites and faecal nitrogen excretion in growing pigs. Animal 6:603-611.

Kavanagh S, Lynch P B, Mara F O, Caffrey P J. 2001. A comparison of total collection and marker technique for the measurement of apparent digestibility of diets for growing pigs. Animal Feed Science and Technology 89: 49-58.Li A, Jiang X, Wang Y, Zhang L, Zhang H, Mehmood K, Li Z, Waqas M, Li J . 2019. The impact of Bacillus subtilis 18 isolated from Tibetan yaks on growth performance and gut microbial community in mice. Microb Pathog 128:153-161.

Liu CF, Zhang LZ, Fu HB, Li WJ, Zhang H, Li JY, Pi L, Zhang YM. 2019. Relationship research between fecal microbes and short chain fatty acid between wild yak and domestic yak. Acta Theriologica Sinica 39:1-7

Peer) reviewing PDF | (2020:01:44574:1:1:NEW 29 Feb 2020) 
500 Li H, Li T, Tu B, Kou Y, and Li X. 2017. Host species shapes the co-occurrence patterns rather

501 than diversity of stomach bacterial communities in pikas. Appl Microbiol Biotechnol $502 \quad 101: 5519-5529$.

503 Li H, Li T, Yao M, Li J, Zhang S, Wirth S, Cao W, Lin Q, Li X. 2016. Pika Gut May Select 504 for Rare but Diverse Environmental Bacteria. Front Microbiol 7:1269.

505 Liu X, Fan H, Ding X, Hong Z, Nei Y, Liu Z, Li G, Guo H.2014. Analysis of the gut microbiota 506 by high-throughput sequencing of the V5-V6 regions of the 16S rRNA gene in donkey. $507 \quad$ Curr Microbiol 68:657-662.

508 Magoc T, Salzberg SL. 2011. FLASH: fast length adjustment of short reads to improve genome 509 assemblies. Bioinformatics 27(21):2957-2963.

510 Mariat D Firmesse O, Levenez F, Guimaraes V, Sokol H, Dore J, Corthier G, Furet JP. 2009. The Firmicutes/Bacteroidetes ratio of the human microbiota changes with age. $B M C$ Microbiol 9:123.

513

Middelbos IS, Vester Boler BM, Qu A, White BA, Swanson KS, Fahey GC, Jr. 2010. Phylogenetic characterization of fecal microbial communities of dogs fed diets with or without supplemental dietary fiber using 454 pyrosequencing. PLoS One 5:e9768.

Moehlman PDR. 2002. Equids: zebras, asses, and horses: status survey and conservation action plan: IUCN.

O'Hara AM, Shanahan F. 2006. The gut flora as a forgotten organ. EMBO Rep 7:688-693.

Paul B. Eckburg EMB, Charles N Bernstein, Elizabeth Purdom, Les Dethlefsen, Michael Human Intestinal Microbial Flora. SCIENCE 308 (5728):1635-1638. 
522 Podgornaia AI, and Laub MT. 2013. Determinants of specificity in two-component signal 523 transduction. Curr Opin Microbiol 16:156-162.

524 Pruesse E, Quast C, Knittel K, Fuchs BM, Ludwig W, Peplies J, Glockner FO. 2007. SILVA:

525 a comprehensive online resource for quality checked and aligned ribosomal RNA sequence 526 data compatible with ARB. Nucleic Acids Res 35:7188-7196.

527 Qin J, Li Y, Cai Z, Li S, Zhu J, Zhang F, Liang S, Zhang W, Guan Y, Shen D, Peng Y, Zhang 528 D, Jie Z, Wu W, Qin Y, Xue W, Li J, Han L, Lu D, Wu P, Dai Y, Sun X, Li Z, Tang 529

530

531

532

533

534

535

536

537

538

539

540

541

542

543 A, Zhong S, Li X, Chen W, Xu R, Wang M, Feng Q, Gong M, Yu J, Zhang Y, Zhang M, Hansen T, Sanchez G, Raes J, Falony G, Okuda S, Almeida M, LeChatelier E, Renault P, Pons N, Batto JM, Zhang Z, Chen H, Yang R, Zheng W, Li S, Yang H, Wang J, Ehrlich SD, Nielsen R, Pedersen O, Kristiansen K, Wang J. 2012. A metagenome-wide association study of gut microbiota in type 2 diabetes. Nature 490:5560.

Ruth E. Ley PJT, Samuel Klein, Gordon JI. 2006. Human gut microbes associated with obesity. Nature 444:1022-1023.

Sahu NP, and Kamra DN. 2002. Microbial Eco-system of the Gastro-intestinal Tract of Wild Herbivorous Animals. Journal of Applied Animal Research 21:207-230.

Schloss PD, Westcott SL, Ryabin T, Hall JR, Hartmann M, Hollister EB, Lesniewski RA, Oakley BB, Parks DH, Robinson CJ, Sahl JW, Stres B, Thallinger GG, Van Horn DJ, Weber CF. 2009. Introducing mothur: open-source, platform-independent, communitysupported software for describing and comparing microbial communities. Appl Environ Microbiol 75:7537-7541. 
544 Sekirov I, Russell SL, Antunes LC, and Finlay BB. 2010. Gut microbiota in health and disease.

545 Physiol Rev 90:859-904. doi:10.1152/physrev.00045.2009.

546 Shannon P, Markiel A, Ozier O, Baliga NS, Wang JT, Ramage D, Amin N, Schwikowski B,

547

548

549

550

551

552

553

554

555

556

557

558

559

560

561

562

563

564

Ideker T. 2003. Cytoscape: a software environment for integrated models of biomolecular interaction networks. Genome research 13:2498-2504.

Shepherd ML, Swecker WS, Jr Jensen RV, Ponder MA. 2012 Characterization of the fecal bacteria communities of forage-fed horses by pyrosequencing of $16 \mathrm{~S}$ rRNA V4 gene amplicons. FEMS Microbiol Lett 326:62-68.

Soest PJV, Robertson JB, and Lewis BA. 1991. Methods for Dietary Fiber, Neutral Detergent Fiber, and Nonstarch Polysaccharides in Relation to Animal Nutrition. Journal of Dairy Science 74:3583-3597.

Sun MY, Dafforn KA, Johnston EL, Brown MV. 2013. Core sediment bacteria drive community response to anthropogenic contamination over multiple environmental gradients. Environ Microbiol 15:2517-2531.

Schwiertz A, Taras D, Schäfer K, Beijer S, Bos NA, Donus C, and Hardt PD. 2010. Microbiota and SCFA in lean and overweight healthy subjects. Obesity 18:190-195.

Turnbaugh PJ, Ley RE, Mahowald MA, Magrini V, Mardis ER, Gordon JI. 2006. An obesityassociated gut microbiome with increased capacity for energy harvest. Nature 444:10271031.

Van Keulen J, Young BA. 1997. Evaluation of acid-insoluble ash as a natural marker in ruminant digestibilitystudies.J. Anim. Sci. 44: 282-287. 
565 Wang Q, Garrity GM, Tiedje JM, Cole JR. 2007. Naive Bayesian classifier for rapid assignment

566 of rRNA sequences into the new bacterial taxonomy. Appl Environ Microbiol 73:5261$567 \quad 5267$.

568 Wardwell LH, Huttenhower C, Garrett WS. 2011. Current concepts of the intestinal microbiota 569 and the pathogenesis of infection. Curr Infect Dis Rep 13:28-34.

570 Wu X, Zhang H, Chen J, Shang S, Wei Q, Yan J, Tu X. 2016. Comparison of the fecal 571 microbiota of dholes high-throughput Illumina sequencing of the V3-V4 region of the 16S

572 rRNA gene. Appl Microbiol Biotechnol

573 Xiong J, Zhu J, Wang K, Wang X, Ye X, Liu L, Zhao Q, Hou M, Qiuqian L, Zhang D. 2014. 574 The temporal scaling of bacterioplankton composition: high turnover and predictability during shrimp cultivation. Microbial ecology 67:256-264.

Xue D, Chen H, Zhao X, Xu S, Hu L, Xu T, Jiang L, Zhan W. 2017. Rumen prokaryotic communities of ruminants under different feeding paradigms on the Qinghai-Tibetan Plateau. Syst Appl Microbiol 40:227-236.

Zeng S, Huang Z, Hou D, Liu J, Weng S, He J. 2017. Composition, diversity and function of intestinal microbiota in pacific white shrimp (Litopenaeus vannamei) at different culture stages. PeerJ 5:e3986.

Zhao JX, Zhang RS, Zhao YL, Du GF, Ma ZY, Jiao JF. 2016. Analysis of cecum bacteria 583 diversity in Xinjiang Donkey. Grass-Feeding Livestock 4:20-25.

Zhang LL, Wei XB, Li ZX, Hu QY, Si DY, Zhang RJ. 2018. The role of gut Bacteria of 585 monogastric animals in amino acid metabolism. Chinese Journal of Animal Science 54

586 (11):17-20.

Zheng S, Gao X. 2000. Status of wild ass in China. Chinese Biodiversity 8:81-87. 
588 Zhou J, Deng Y, Luo F, He Z, Yang Y. 2011. Phylogenetic molecular ecological network of soil microbial communities in response to elevated CO2. MBio 2(4):e0122-11. 
Figure 1

The statistical significance of alpha diversity estimators between TWAs and NPDDs.

TWA=Tibetan wild asses, NPDD=natural pasture domestic donkeys. NS means no significant difference.
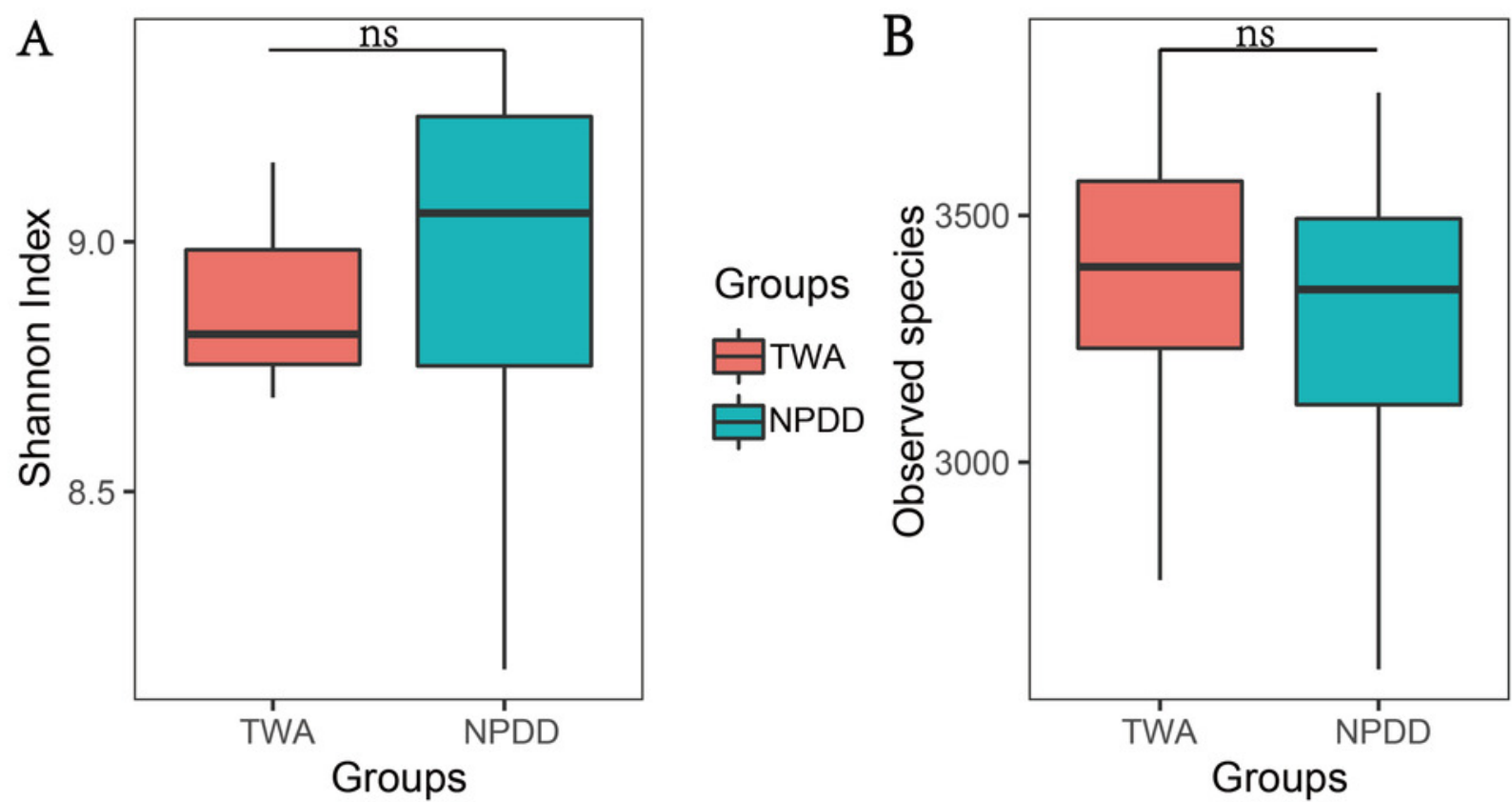

Groups
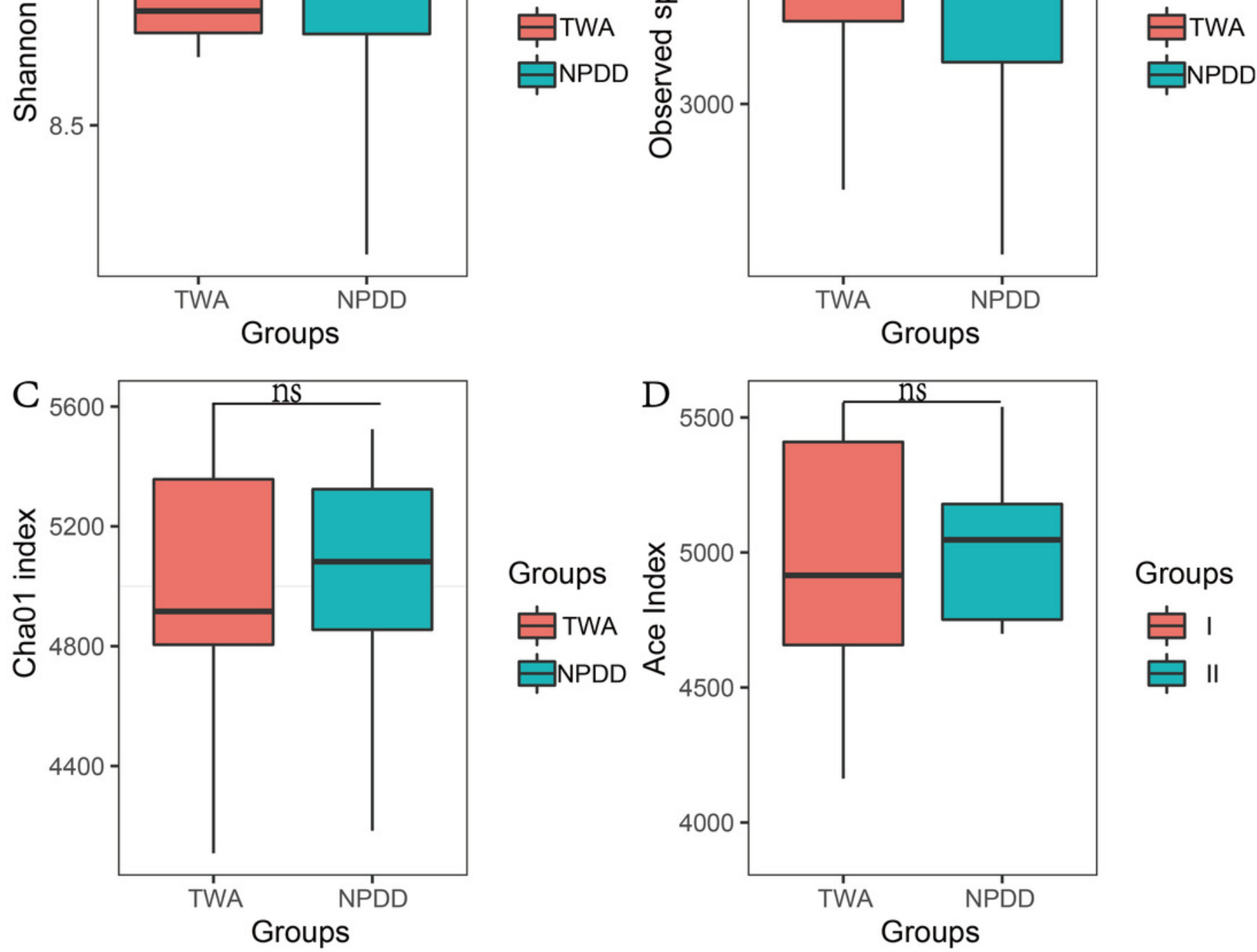
Figure 2

Bacterial community relative abundance at the phylum level of each group.

(A) Bacterial community abundance bar plot at the phylum level. (B) Significance of the top 10 bacterial community abundance percentages at the phylum level. Each phylum that shares annotations was significantly different $(P<0.05)$. The error bar meant the value of standard error. TWA=Tibetan wild asses, NPDD=natural pasture domestic donkeys. 


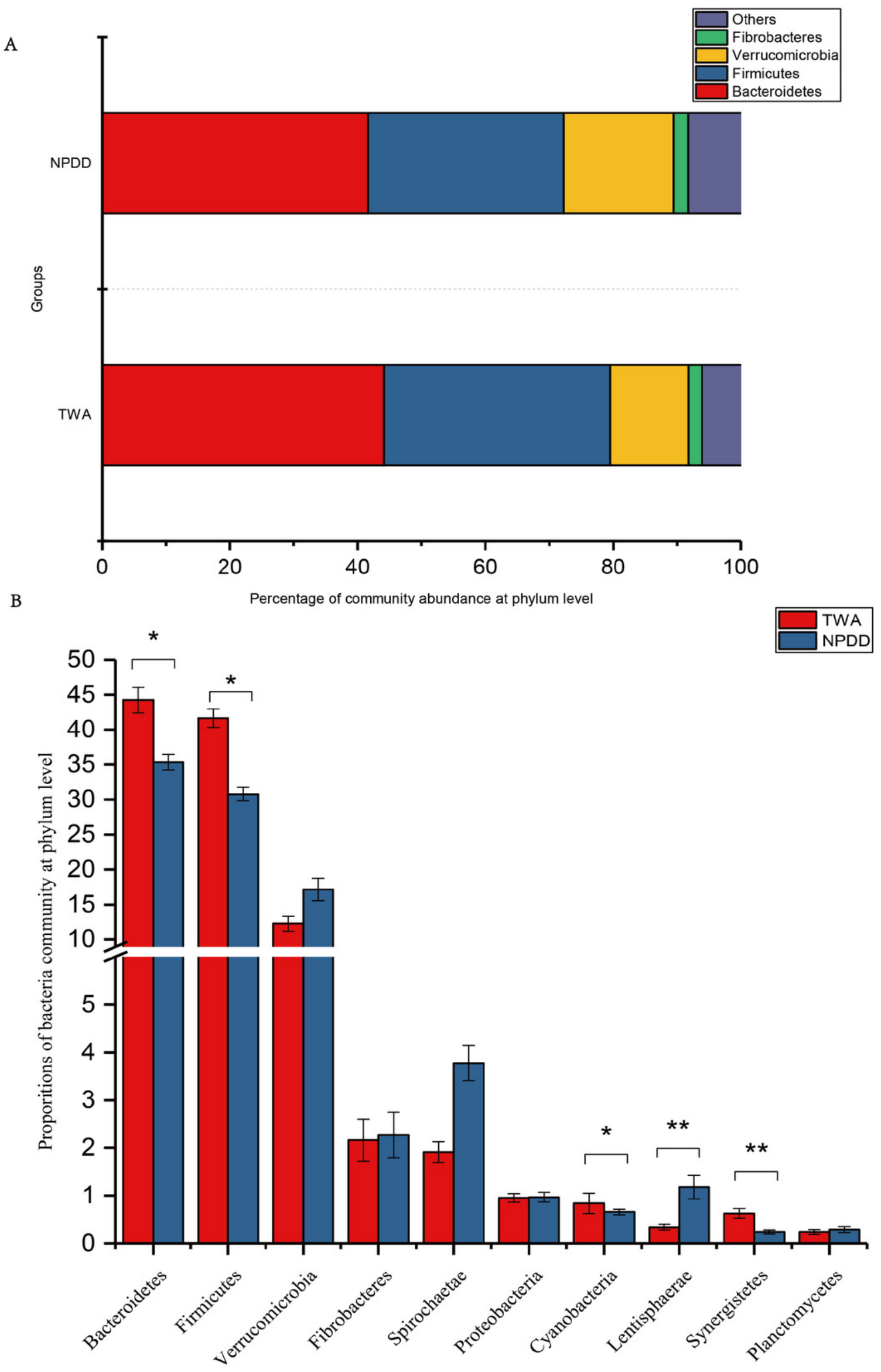




\section{Figure 3}

Bacterial community abundance at the genus level.

(A) Bacterial community bar plot at the genus level. (B) Statistical significance of the top 15 bacterial community abundance percentages at the genus level. The error bar meant the value of standard error. TWA=Tibetan wild asses, NPDD=natural pasture domestic donkeys. 
A
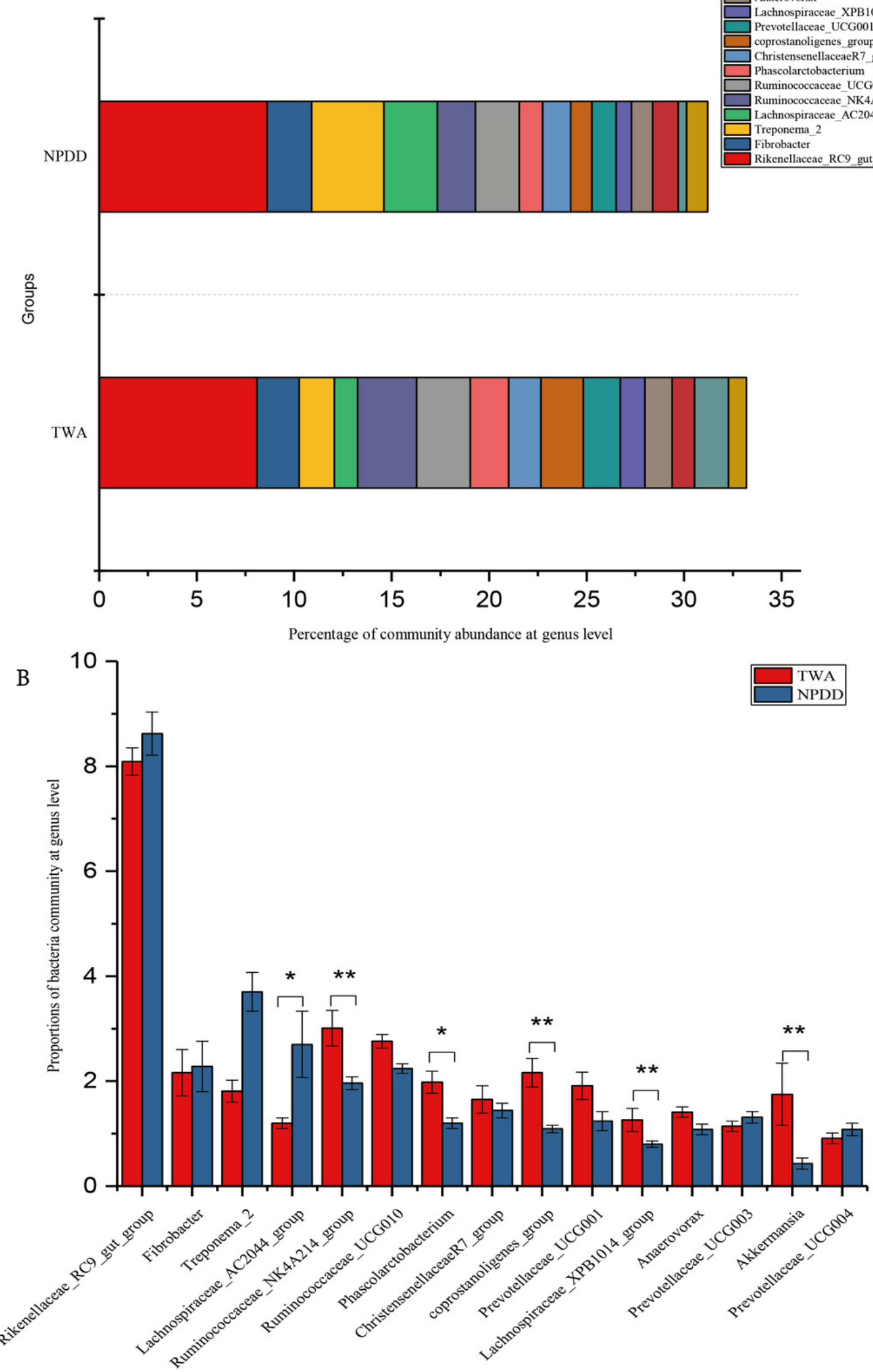

PeerJ reviewing PDF | (2020:01:44574:1:1:NEW 29 Feb 2020) 


\section{Figure 4}

Metabolic pathway prediction at various KEGG levels between TWAs and NPDDs.

(A) The six types of biological functional pathways in KEGG level 1. (B) The 17 significant metabolic pathways in KEGG level 2. The "p-value (corrected)" is the false discovery rate (FDR) value. TWA=Tibetan wild asses, NPDD=natural pasture domestic donkeys. 
A

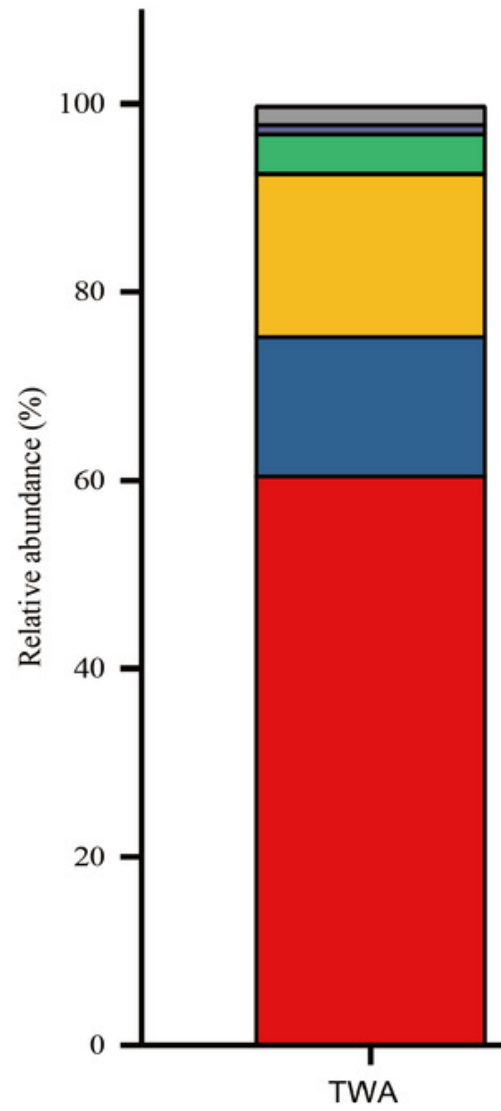

Human Diseases

Organismal Systems

Cellular Processes

Environmental Information Processing

Genetic Information Processing

Metabolism
Amino Acid Metabolism Membrane Transport Substance Dependence | Cell Motility Xenobiotics Biodegradation and Metabolism Cardiovascular Diseases | Endocrine System Energy Metabolism Immune Diseases | Circulatory System | Cancers | Excretory System | Cell Communication | Neurodegenerative Diseases | Signaling Molecules and Interaction | Glycan Biosynthesis and Metabolism Nervous System | Biosynthesis of Other Secondary Metabolites $日$

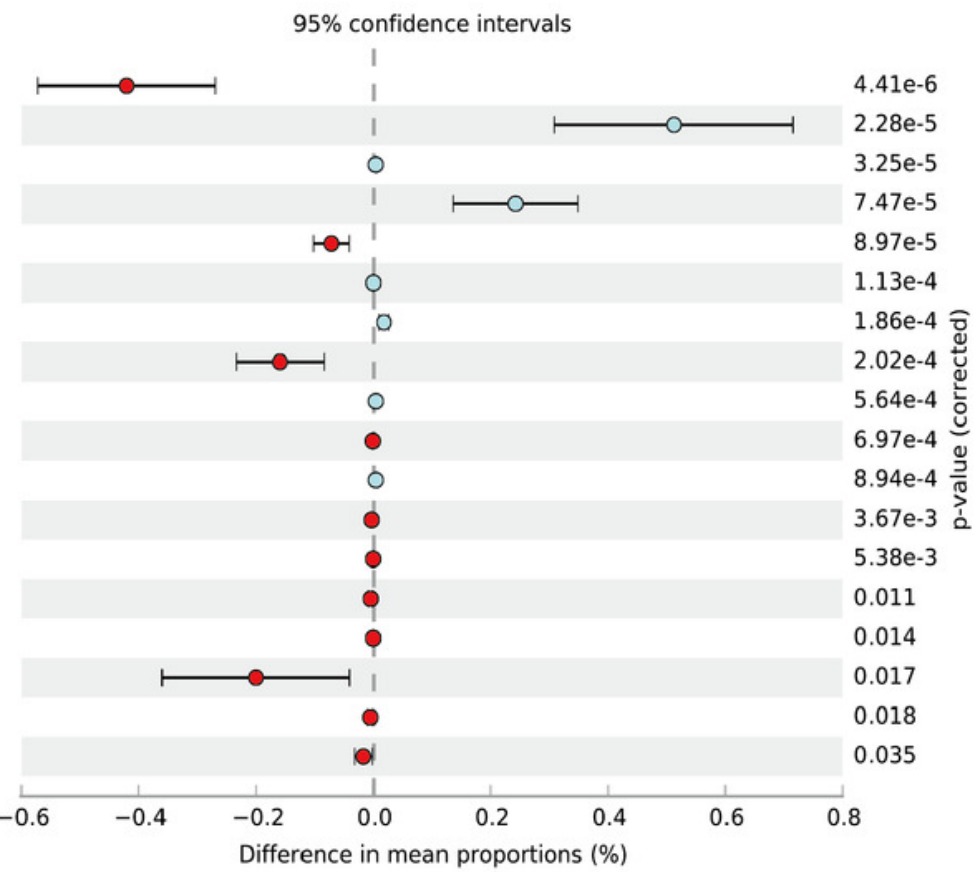


Figure 5

The principal component analysis (PCA) and partial least squares discriminant analysis (PLS-DA) of metabolite pathways between TWAs and NPDDs.

TWA=Tibetan wild asses, NPDD=natural pasture domestic donkeys.

A

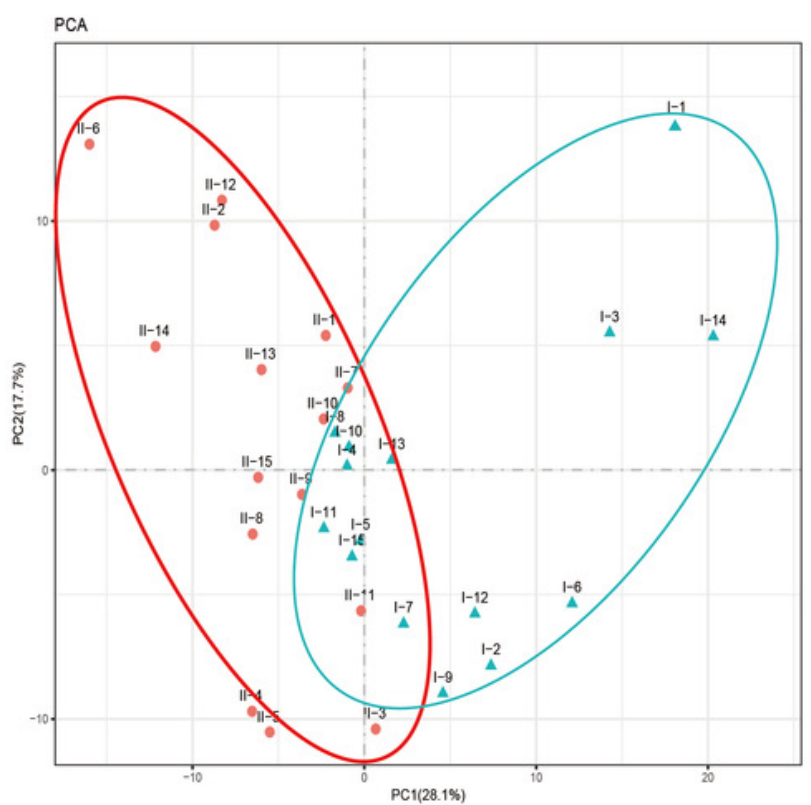

B

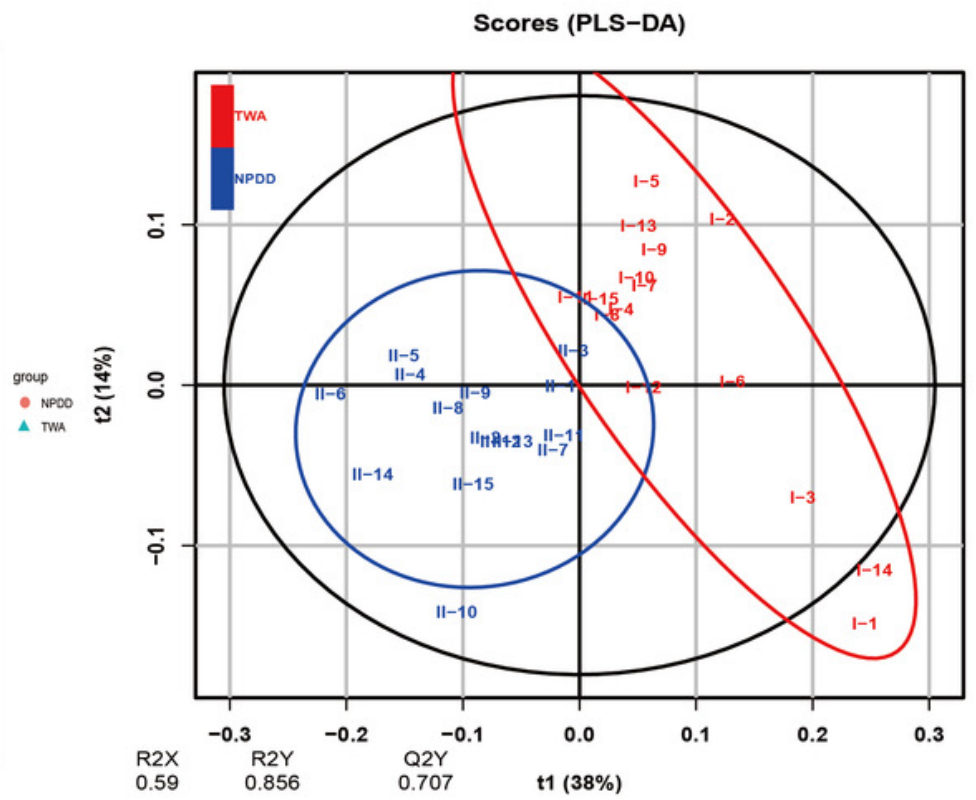


Figure 6

Comparision of gut bacterial networks between TWAs and NPDDs.

(A) The gut bacteria of TWA. (B) The gut bacteria of NPDD. Highly positive correlations are indicated by red color and negative correlations by gray color. TWA=Tibetan wild asses, NPDD=natural pasture domestic donkeys.

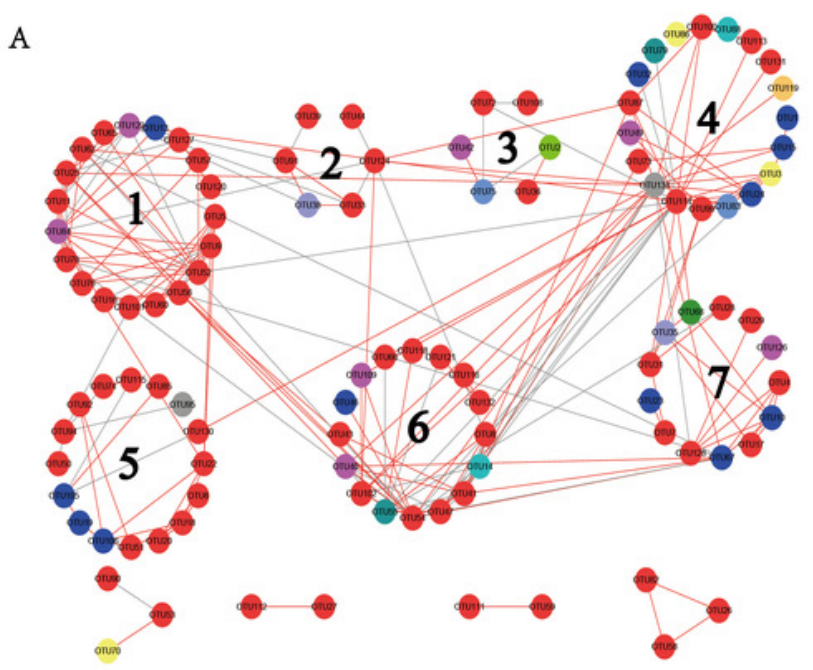

B

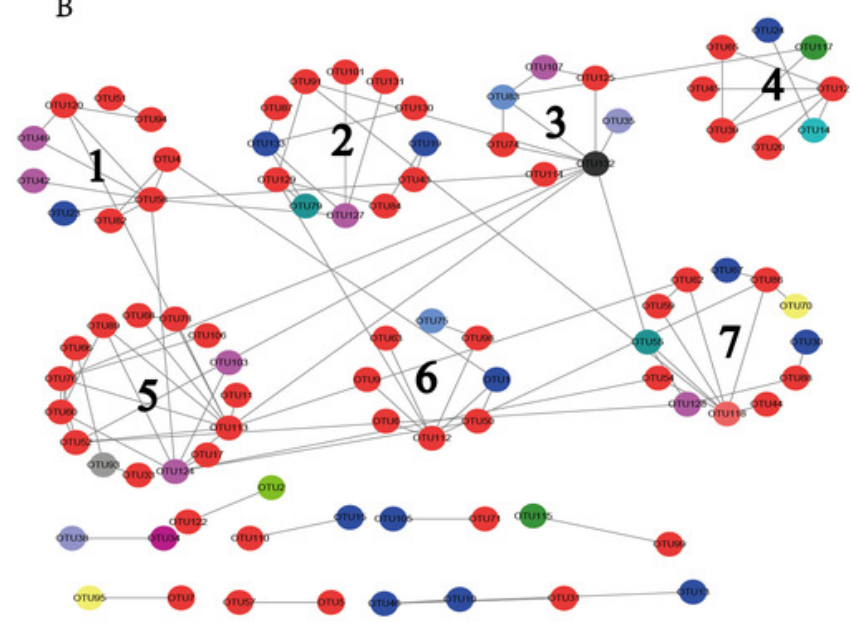

\begin{tabular}{lcc}
\hline Network Indexes & NPDD & TWA \\
\hline No.of samples & 15 & 15 \\
RMT cutoff & 0.76 & 0.76 \\
Total nodes & 89 & 105 \\
Total links & 104 & 207 \\
Positive links & 49 & 149 \\
Negative links & 55 & 58 \\
Average degree (avgK) & 2.34 & 3.94 \\
Average clustering coefficient (avgCC) & 0.12 & 0.27 \\
Centralization of betweeness (CB) & 0.235 & 0.305 \\
Density (D) & 0.027 & 0.038 \\
\hline
\end{tabular}

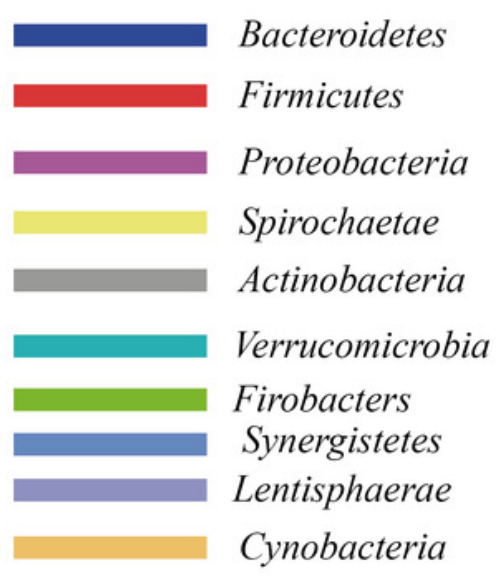




\section{Table $\mathbf{1}$ (on next page)}

Comparison of the nutrient contents in the herbage composition of the TWAs and NPDDs groups.

${ }^{a}$ TWAs refers to Tibetan wild asses, NPDDs refers to natural pasture domestic donkeys. ${ }^{b} \mathrm{EE}$ $=$ ether extract, $\mathrm{CP}=$ crude protein, $\mathrm{ADF}=$ acid detergent fiber, $\mathrm{NDF}=$ neutral detergent fiber, NFC $=$ Non-fibrous carbohydrate. Values in the same row with different superscripts are significantly different $(P<0.05)$. 
1

3

\begin{tabular}{lccc}
\hline Nutrient content of & \multicolumn{2}{c}{ Groups $^{\mathrm{a}}$} & \multirow{2}{*}{ P value } \\
Herbage (\%) & $1.40 \pm 0.10$ & $1.32 \pm 0.02$ & 0.09 \\
\cline { 2 - 3 } $\mathrm{EE}^{\mathrm{b}}$ & $11.13 \pm 1.02$ & $10.96 \pm 0.43$ & 0.09 \\
$\mathrm{CP}$ & $22.51 \pm 1.02$ & $28.50 \pm 1.30$ & 0.76 \\
$\mathrm{ADF}$ & $44.56 \pm 3.06$ & $48.82 \pm 1.87$ & 0.44 \\
$\mathrm{NDF}$ & $27.80 \pm 3.00$ & $25.39 \pm 2.02$ & 0.54 \\
$\mathrm{NFC}$ & $15.12 \pm 1.45^{\mathrm{a}}$ & $13.54 \pm 0.34^{\mathrm{b}}$ & 0.03 \\
Ash & Kobresia & Kobresia & \\
& tibetica, & humilis, & \\
Dominant species of & Kobresia & Kobresia & \\
herbages & pygmaea & capillifolia & \\
& & &
\end{tabular}




\section{Table 2 (on next page)}

The dry matter digestion between TWAs and NPDDs.

TWAs refers to Tibetan wild asses, NPDDs refers to natural pasture domestic donkeys. AIA= Acid-insoluble ash. 
1

\begin{tabular}{cccc}
\hline \multirow{2}{*}{ Indexes } & \multicolumn{2}{c}{ Groups } & \multirow{2}{*}{$P$ value } \\
\cline { 2 - 3 } & TWAs & NPDDs & \\
\hline AIA in faces & $0.41 \pm 0.05^{\mathrm{b}}$ & $0.47 \pm 0.01^{\mathrm{a}}$ & 0.000 \\
AIA in herbage & $0.17 \pm 0.04^{\mathrm{b}}$ & $0.24 \pm 0.02^{\mathrm{a}}$ & 0.274 \\
Dry matter digestibility & $58.90 \pm 8.21^{\mathrm{a}}$ & $50.46 \pm 3.71^{\mathrm{b}}$ & 0.043 \\
\hline
\end{tabular}




\section{Table 3 (on next page)}

The taxonomic identification of putative keystone genus in network of TWAs and NPDDs bacterial community.

Fir Firmicutes, Act Actinobacteria, Cya, Cyanobacteria. TWAs refers to Tibetan wild asses, NPDDs refers to natural pasture domestic donkeys. ${ }^{a}$ The average relative abundance of the OTUs in TWAs and NPDDs. ${ }^{b}$ The abundance ranking of the OTU in TWAs and NPDDs in TWAs and NPDDs according to order from high to low. 


\section{Average}

Species OTUID relative
Taxnomy
Abundance

Node type $\quad \mathrm{Pi} \quad \mathrm{Zi}$

Abundance ${ }^{a}$

TWAs

OTU67

0.0662

OTU114 0.0046

Unclassified

OTU124 $\quad 0.0034$

Oscillospira (Fir)

OTU134 $\quad 0.0027$

Arthrobacter (Act)

Cellulosilyticum (Fir)

Erysipelatoclostridium

OTU112 0.0035

OTU118 0.0023

OTU54

0.0517

OTU114

0.0032

(Fir)

Oscillatoria (Cya)

Anaerovibrio (Fir)

Unclassified

126th Module hubs $\quad 0.4490 \quad 2.5019$

137 th

Module hubs

$0.2449 \quad 2.9542$

68th

Connector

$0.6667 \quad 0.8452$

130th

Connector

$0.6400 \quad 2.1243$ 